Supplement of The Cryosphere, 13, 2133-2148, 2019

https://doi.org/10.5194/tc-13-2133-2019-supplement

(C) Author(s) 2019. This work is distributed under

the Creative Commons Attribution 4.0 License.

(c) (1)

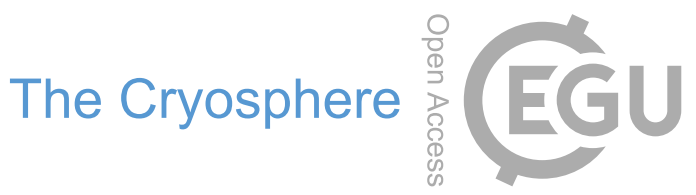

Supplement of

\title{
Eemian Greenland ice sheet simulated with a higher-order model shows strong sensitivity to surface mass balance forcing
}

Andreas Plach et al.

Correspondence to: Andreas Plach (andreas.plach@uib.no)

The copyright of individual parts of the supplement might differ from the CC BY 4.0 License. 


\section{Additional simulations using the shallow shelf approximation (SSA)}

In addition to the computationally costly $3 \mathrm{D}$ higher-order simulations, we carried out a series of simulations with a computationally efficient 2D SSA model for a more comprehensive study of the parameter space. The range of 2D SSA experiments was used to select the parameters for the 3D higherorder simulations discussed in the manuscript. A list of the 2D SSA experiments is shown in Table S1 where experiments with different basal friction as in the manuscript are highlighted with gray shading. The $2 \mathrm{D}$ control and 2D SMB experiments in Table S1 are discussed as the ice flow experiments in the manuscript to illustrate the differences resulting from using the 3D higher-order compared to the 2D SSA ice flow approximation.

The additional, more extreme experiments $(\geq 20 \%$ change of basal friction) show unrealistically large changes in ice surface elevation at most ice core locations (red (MAR-SEB) and blue (MAR-BESSI) lines in Fig. S1). While changes of $10 \%$ of the basal friction in the basal experiments (as discussed in the manuscript; solid light orange lines in Fig. S1d)) result in changes of ice surface elevation which are still within the NEEM surface change reconstructions (gray shading in Fig. S1d), the $\geq 20 \%$ change experiments (red lines in Fig. S1d) lead to changes outside of these NEEM reconstructions.

Additionally, we illustrate the evolution of the ice volume in the 2D SSA experiments (Fig. S2) and the consequences of $\geq 20 \%$ change to the basal friction on the evolution of the ice volume (red (MAR-SEB) and blue (MARBESSI) lines in Fig. S2). 
Table S1: Overview of SSA experiments

\begin{tabular}{|c|c|c|c|}
\hline type of experiment & SMB method & basal friction & ice flow approx. \\
\hline $2 \mathrm{D}$ control & MAR-SEB & modern & 2D SSA \\
\hline $2 \mathrm{D} S M B$ & MAR-BESSI & modern & 2D SSA \\
\hline 2D basal (reduced) & MAR-SEB & $0.5 *$ modern (entire ice sheet) & 2D SSA \\
\hline 2D basal (reduced) & MAR-BESSI & $0.5 *$ modern (entire ice sheet) & 2D SSA \\
\hline 2D basal (reduced) & MAR-SEB & $0.8 *$ modern (entire ice sheet) & 2D SSA \\
\hline 2D basal (reduced) & MAR-BESSI & $0.8 *$ modern (entire ice sheet) & 2D SSA \\
\hline 2D basal (reduced) & MAR-SEB & $0.9 *$ modern (entire ice sheet) & 2D SSA \\
\hline 2D basal (reduced) & MAR-BESSI & $0.9 *$ modern (entire ice sheet) & 2D SSA \\
\hline 2D basal (enhanced) & MAR-SEB & $1.1 *$ modern (entire ice sheet) & 2D SSA \\
\hline 2D basal (enhanced) & MAR-BESSI & $1.1 *$ modern (entire ice sheet) & 2D SSA \\
\hline 2D basal (enhanced) & MAR-SEB & $1.2 *$ modern (entire ice sheet) & 2D SSA \\
\hline 2D basal (enhanced) & MAR-BESSI & $1.2 *$ modern (entire ice sheet) & 2D SSA \\
\hline 2D basal (enhanced) & MAR-SEB & $2.0 *$ modern (entire ice sheet) & 2D SSA \\
\hline 2D basal (enhanced) & MAR-BESSI & $2.0 *$ modern (entire ice sheet) & 2D SSA \\
\hline 2D outlets (reduced) & MAR-SEB & $0.5 *$ modern (outlet glaciers) & 2D SSA \\
\hline $2 \mathrm{D}$ outlets (reduced) & MAR-BESSI & $0.5 *$ modern (outlet glaciers) & 2D SSA \\
\hline 2D outlets (reduced) & MAR-SEB & $0.8 *$ modern (outlet glaciers) & 2D SSA \\
\hline 2D outlets (reduced) & MAR-BESSI & $0.8 *$ modern (outlet glaciers) & 2D SSA \\
\hline $2 \mathrm{D}$ outlets (reduced) & MAR-SEB & $0.9 *$ modern (outlet glaciers) & 2D SSA \\
\hline 2D outlets (reduced) & MAR-BESSI & $0.9 *$ modern (outlet glaciers) & 2D SSA \\
\hline $2 \mathrm{D}$ outlets (enhanced) & MAR-SEB & $1.1 *$ modern (outlet glaciers) & 2D SSA \\
\hline $2 \mathrm{D}$ outlets (enhanced) & MAR-BESSI & $1.1 *$ modern (outlet glaciers) & 2D SSA \\
\hline $2 \mathrm{D}$ outlets (enhanced) & MAR-SEB & $1.2 *$ modern (outlet glaciers) & 2D SSA \\
\hline $2 \mathrm{D}$ outlets (enhanced) & MAR-BESSI & $1.2 *$ modern (outlet glaciers) & 2D SSA \\
\hline 2D outlets (enhanced) & MAR-SEB & $2.0 *$ modern (outlet glaciers) & 2D SSA \\
\hline $2 \mathrm{D}$ outlets (enhanced) & MAR-BESSI & $2.0 *$ modern (outlet glaciers) & 2D SSA \\
\hline 2D altitude & MAR-SEB & modern & 2D SSA \\
\hline 2D altitude & MAR-BESSI & modern & 2D SSA \\
\hline $2 \mathrm{D}$ relaxed & MAR-SEB & modern & $2 \mathrm{D} \mathrm{SSA}$ \\
\hline
\end{tabular}

Additional 2D SSA experiments with different parameters as in the manuscript are indicated by gray shading. 

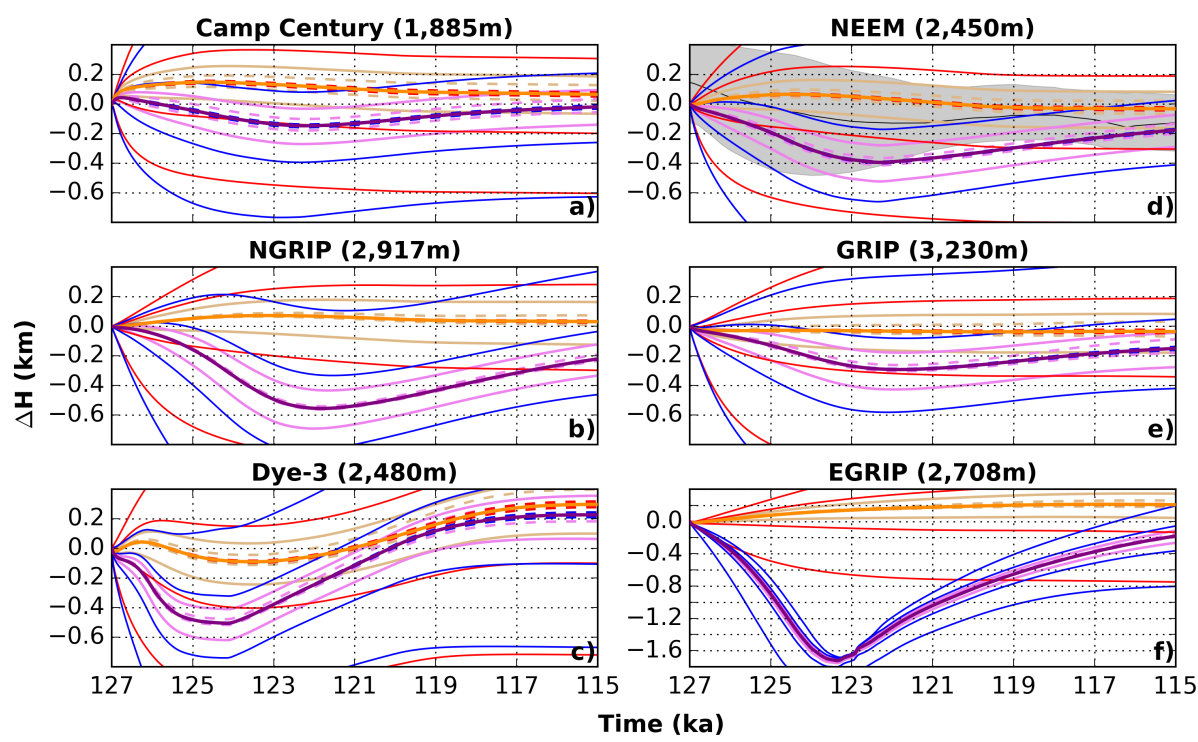

Time (ka)

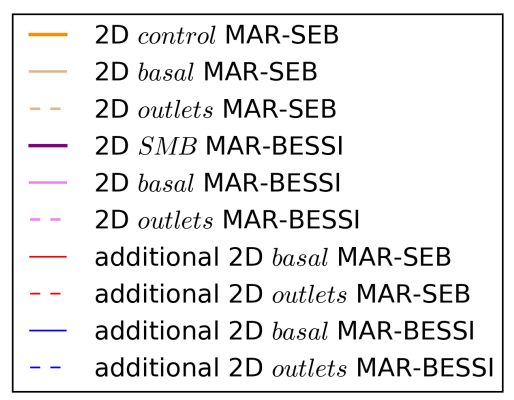

Figure S1: Ice surface evolution at Greenland ice core locations for the 2D control (MAR-SEB, orange, bold) and the 2D SMB (MAR-BESSI, purple, bold) experiments in comparison with the $2 \mathrm{D}$ basal/outlets sensitivity experiments. The $2 \mathrm{D}$ basal (friction $* 0.9 /{ }^{*} 1.1$ for the entire ice sheet) and $2 \mathrm{D}$ outlets sensitivity experiments (friction $*^{*} 0.5 /{ }^{*} 2.0$ at the outlet glaciers) are indicated with thin solid and thin dashed lines, respectively. Additional 2D basal/outlets sensitivity experiments (see Tab. S1) are shown with red (MAR-SEB) and blue (MAR-BESSI) solid/dashed lines. Camp Century, NEEM, NGRIP, GRIP, and Dye-3 are shown on the same scale; EGRIP is shown on a different scale. Surface elevation reconstructions from total gas content at NEEM are indicated with gray shading. 


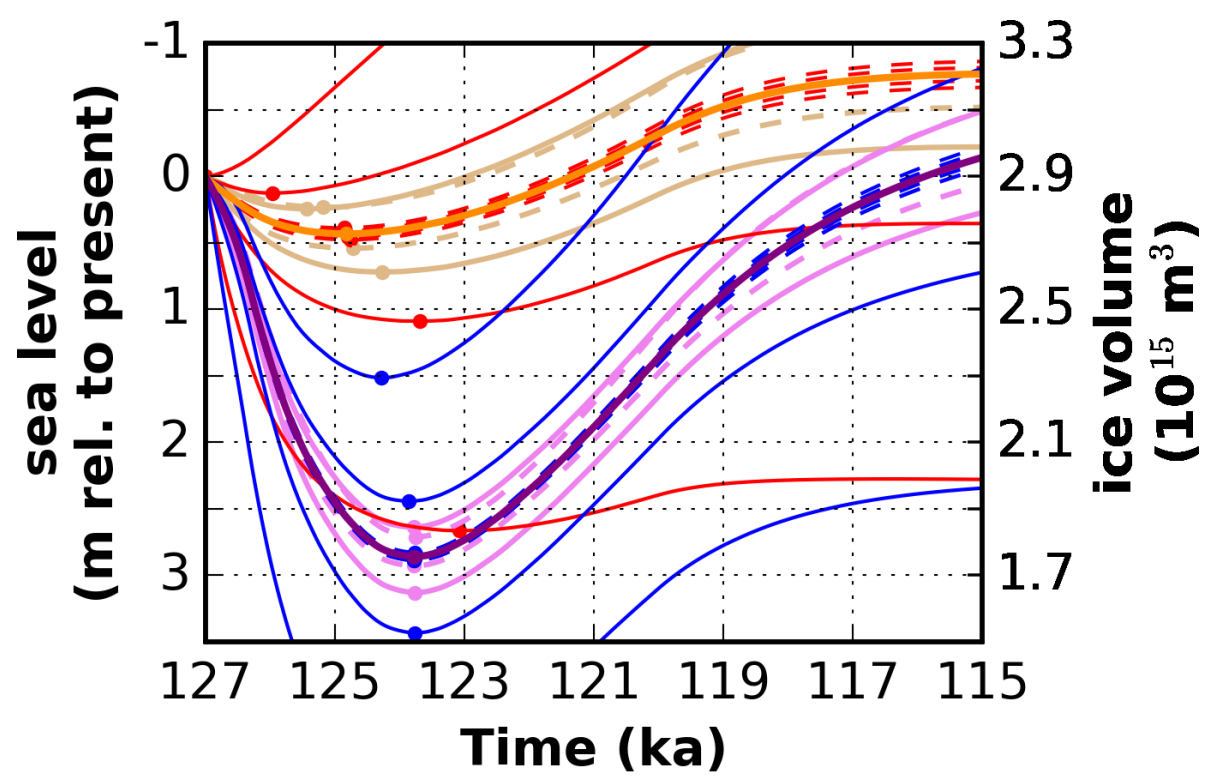

\begin{tabular}{|llll|}
\hline- & 2D control MAR-SEB & - & additional 2D basal MAR-SEB \\
- & 2D basal MAR-SEB & - & additional 2D outlets MAR-SEB \\
- & 2D outlets MAR-SEB & - & additional 2D basal MAR-BESSI \\
- & 2D SMB MAR-BESSI & - & additional 2D outlets MAR-BESSI \\
- & 2D basal MAR-BESSI & - & time of minimum \\
- & 2D outlets MAR-BESSI & & \\
\hline
\end{tabular}

Figure S2: Evolution of the ice volume for the 2D control (MAR-SEB, orange, bold) and the 2D SMB (MAR-BESSI, purple, bold) experiments in comparison with the $2 \mathrm{D}$ basal/outlets sensitivity experiments. The 2D basal (friction ${ }^{*} 0.9 /{ }^{*} 1.1$ for the entire ice sheet) and $2 \mathrm{D}$ outlets sensitivity experiments (friction $* 0.5 / * 2.0$ at the outlet glaciers) are indicated with thin solid and thin dashed lines, respectively. Additional 2D basal/outlets sensitivity experiments (see Tab. S1) are shown with red (MAR-SEB) and blue (MAR-BESSI) solid/dashed lines. Note that the lower friction experiments give lower volumes. The minimum of the respective experiments is indicated with circles. 


\section{Map of basal friction coefficients}

Figure S3 shows the spatial distribution of the basal friction coefficients in the control experiment discussed in the manuscript. The basal friction coefficients are derived from inversion employing modern surface velocities (see Sec. 2.2 in the manuscript). This map of basal frictions coefficients is modified in the basal and outlets experiments.
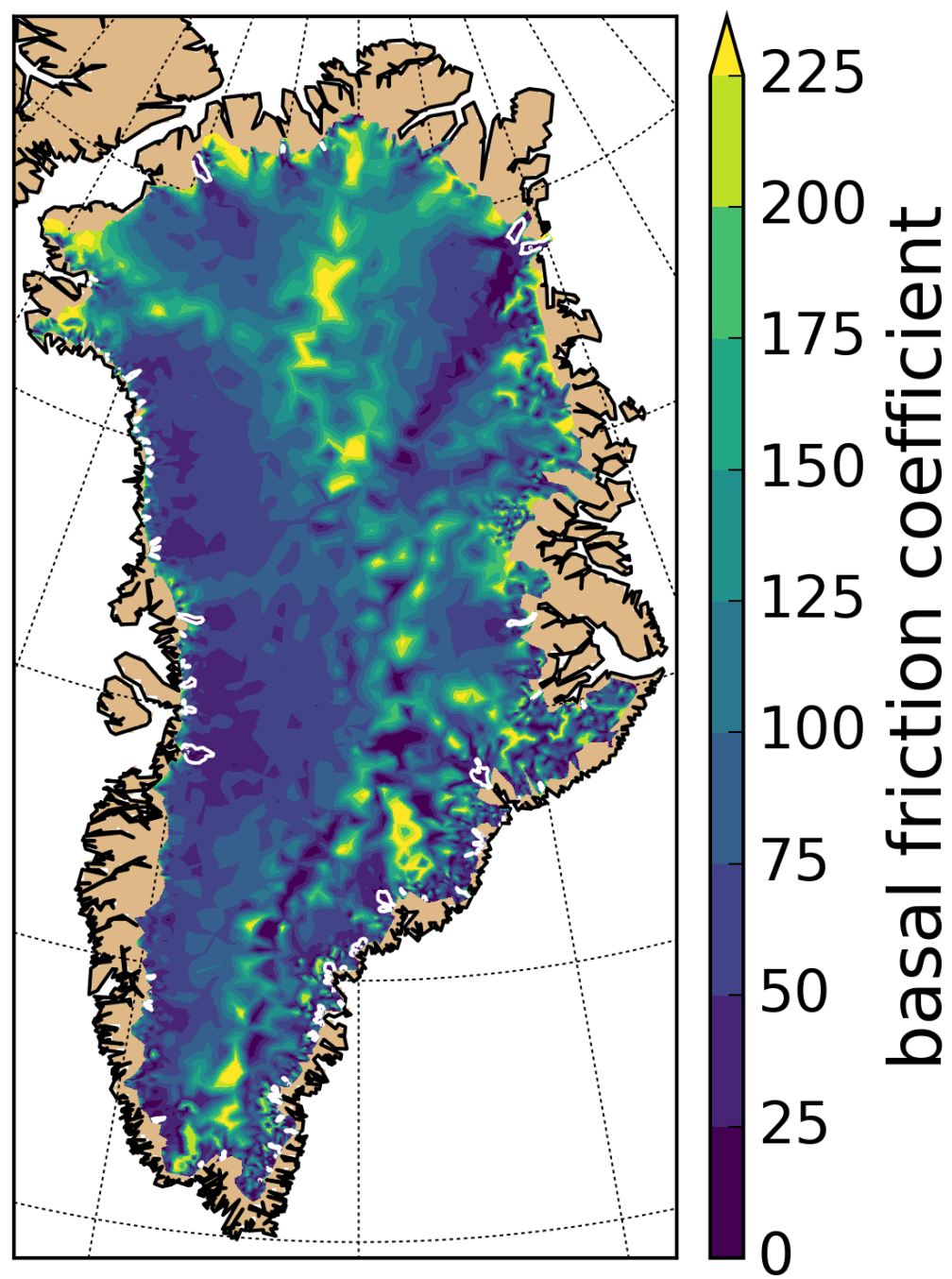

Figure S3: Basal friction coefficients for the control experiment in the manuscript. Regions affected in the outlets experiments are indicated with white contours. 